Published in "Chemistry of Materials 21(11): 2169-2172, 2009"

which should be cited to refer to this work.

\section{Metallosupramolecular Side-Chain Polymers and Polyelectrolyte- Metallosupramolecular Surfactant Complexes}

M. R. Hammond ${ }^{\dagger}$ A. K. Andreopoulou, ${ }^{\ddagger}$ E. Pefkianakis ${ }^{\ddagger}$ J. K. Kallitsis, ${ }^{\ddagger}$ and R. Mezzenga ${ }^{*}, \dagger, \S$

Department of Physics and Fribourg Center for Nanomaterials, University of Fribourg, Chemin du Musée 3, 1700 Fribourg, Switzerland, Department of Chemistry and Institute of Chemical Engineering and High Temperature Chemical Processes, FORTH/ICE-HT, Univeristy of Patras, 26504 Patras, Greece, and Nestlé Research Center, Vers-chez-les-blanc, 1000 Lausanne 26, Switzerland

Metal coordination has been exploited extensively in supramolecular chemistry, ${ }^{1}$ and the creation of supramolecular polymeric materials ${ }^{2}$ via metal-ligand bonds is an active area of research. Side-chain type supramolecular polymers are of interest because of the ability of these materials to form liquid-crystalline morphologies, with instances of such materials being created via hydrogen bonding, ionic bonding, metal complexation, and combinations thereof. ${ }^{3,4}$ Indeed, the pace of research on polymers with side-chains attached by metal coordination is accelera-

* Corresponding author. E-mail: raffaele.mezzenga@unifr.ch.

University of Fribourg.

* University of Patras.

$\$$ Nestlé Research Center.

(1) Lehn, J. M. Angew. Chem., Int. Ed. 1988, 27, 89-112.

(2) Ikkala, O.; ten Brinke, G. Science 2002, 295, 2407-2409.

(3) Hammond, M. R.; Mezzenga, R. Soft Matter 2008, 4, 952-961.

(4) Pollino, J. M.; Weck, M. Chem. Soc. Rev. 2005, 34, 193-207. ting. ${ }^{5-9}$ In 2005, several of us published the syntheses of metallosupramolecular polymers (MSPs) bearing alkyl-chain decorated dendrons attached to main chains via a terpyridineRu-terpyridine $\left(\mathrm{Ru}(\mathrm{tpy})_{2}\right)$ complexation strategy. ${ }^{7,8}$ More recently, Aamer and Tew reported the synthesis and morphological characterization of two very similar MSPs, ${ }^{9}$ also based on polymers bearing pendant tpy groups. The first, in which the polymer was complexed with a simple terpyridine group, showed no mesomorphic structure, whereas the second, in which a C16 alkyl tail was attached to the supramolecularly bound terpyridine group, was shown to selforganize into a hexagonal columnar morphology, with some crystallization occurring between the C16 tails. This latter material was also shown to display nematic lyotropic behavior in concentrated solution.

In general, it has been well-documented over the last decades that alkoxy tails can induce mesomorphous features in polymeric structures that do not inherently self-assemble. Such alkoxy groups can be directly attached onto polymeric main backbones or indirectly through the dendronization of flexible, rigid-flexible or even fully aromatic main chains with periphery alkoxy-decorated dendrons. ${ }^{10-18}$

(5) Valkama, S.; Lehtonen, O.; Lappalainen, K.; Kosonen, H.; Castro, P.; Repo, T.; Torkkeli, M.; Serimaa, R.; ten Brinke, G.; Leskelä, M.; Ikkala, O. Macromol. Rapid Commun. 2003, 24, 556-560.

(6) Pollino, J. M.; Stubbs, L. P.; Weck, M. Macromolecules 2003, 36, 2230-2234.

(7) Andreopoulou, A. K.; Kallitsis, J. K. Eur. J. Org. Chem. 2005, 20, 4448-4458

(8) Tzanetos, N. P.; Andreopoulou, A. K.; Kallitsis, J. K. J. Polym. Sci. Polym. Chem. 2005, 43, 4838-4848.

(9) Aamer, K. A.; Tew, G. N. Macromolecules 2007, 40, 2737-2744.

(10) Ballauff, M. Makromol. Chem. Rapid Commun. 1986, 7, 407-414.

(11) Rodriguez-Parada, J. M.; Duran, R.; Wegner, G. Macromolecules 1989, $22,2507-2516$

(12) Kakali, F.; Kallitsis, J.; Pakula, T.; Wegner, G. Macromolecules 1998, 31, 6190-6198. 
Scheme 1. Chemical Structures of Metallosupramolecular Polymers (MSP) and PAA-Metallosupramolecular Surfactant (PAA(MSS)) Complexes
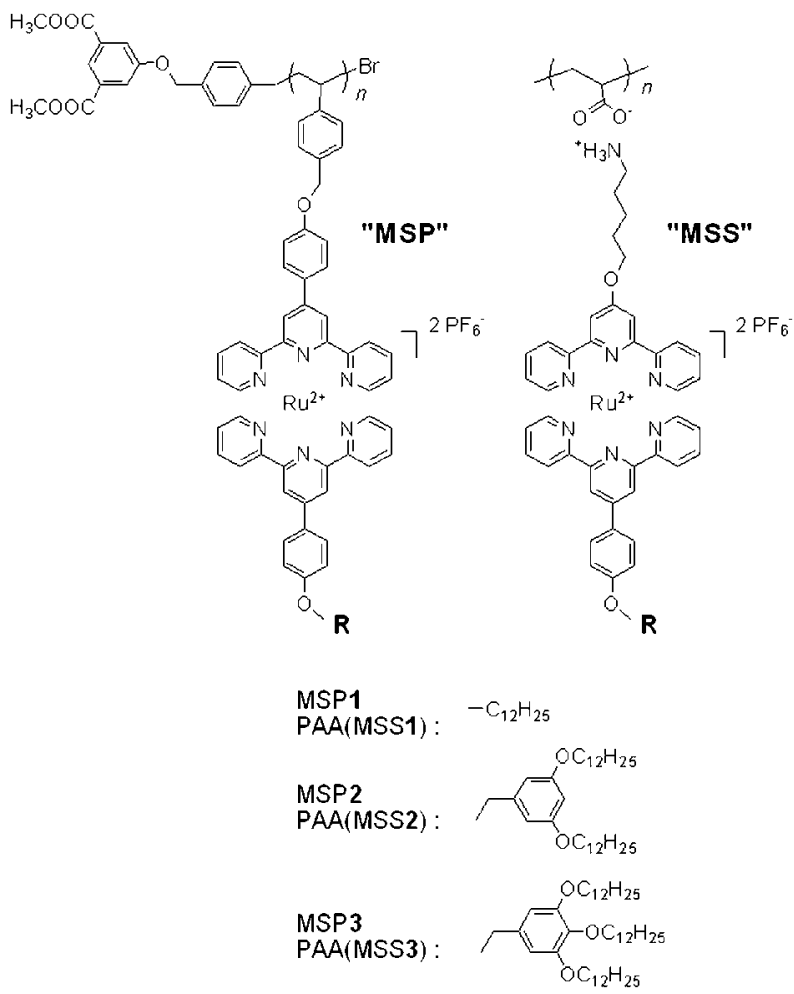

We report here on the mesomorphous structures adopted in the solid state by a homologous set of such MSPs, in which dendrons bearing one, two, and three $\mathrm{C} 12$ alkyl tails are complexed to the polymer backbone; these are MSP1, MSP2, and MSP3, respectively, and their chemical structures are shown in Scheme 1. Similarly to the alkyl tail-bearing material of Aamer and Tew, the resulting mesomorphous structures are presumably due to microphase separation into alkyl tail-rich domains and domains rich in charged and polarizable groups. Further, we show how sequential metal-ligand complexation and ionic complexation can be employed to create polyelectrolyte-metallosupramolecular surfactant (PE-MSS) complexes with chemical structures and morphologies similar to the MSPs. Specifically, we have synthesized and characterized ionic complexes between poly(acrylic acid) (PAA) and the metallosupramolecular surfactants (MSS1, MSS2, and MSS3), shown in Scheme $1 .{ }^{19}$ Morphological characterization was performed using small- and wide-angle X-ray scattering (SAXS and WAXS), and optical birefringence measurements using cross-polarized optical microscopy (CPOM).

(13) Schlüter, A. D.; Rabe, J. P. Angew. Chem., Int. Ed. 2000, 39, 864883.

(14) Tschierske, C. J. Mater. Chem. 2001, 11, 2647-2671.

(15) Andreopoulou, A. K.; Carbonnier, B.; Kallitsis, J. K.; Pakula, T. Macromolecules 2004, 37, 3576-3587.

(16) Percec, V.; Cho, W.-D.; Ungar, G.; Yeardley, D. J. P. Angew. Chem., Int. Ed. 2000, 39, 1597-1602.

(17) Percec, V.; Rudick, J. G.; Peterca, M.; Heiney, P. A. J. Am. Chem. Soc. 2008, 130, 7503-7508.

(18) Rudick, J. G.; Percec, V. Acc. Chem. Res. 2008, 41, 1641-1652.

(19) The syntheses of MSS1 and MSS2 have been recently reported: Stefopoulos, A. A.; Pefkianakis, E. K.; Papagelis, K.; Andreopoulou, A. K.; Kallitsis, J. K. J. Polym. Sci., Polym. Chem. 2009, 47, 25512559.
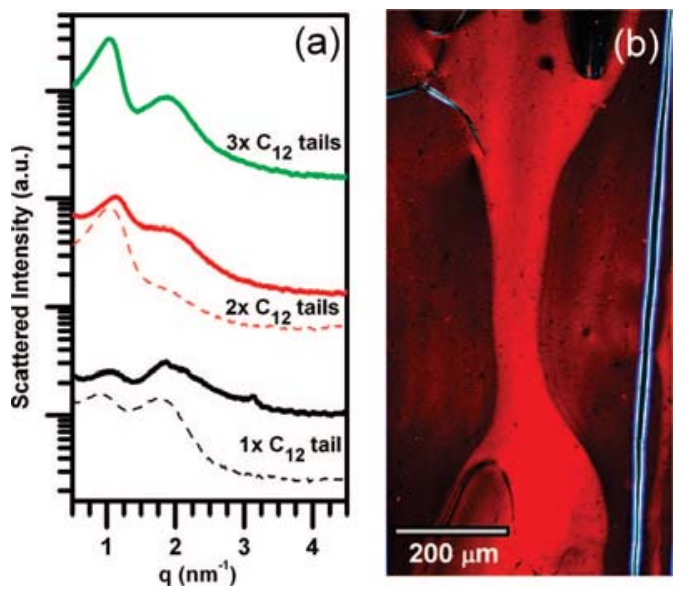

Figure 1. (a) SAXS results for the MSP complexes with 1,2, and $3 \mathrm{C} 12$ alkyl tails (solid curves) and for PAA(MSS) (ionic) complexes with 1 and 2 C12 alkyl tails (dashed curves). (b) Cross-polarized optical microscopy image of a sample of the MSP2 material, prepared by shearing a concentrated MSP2 solution and subsequent solvent removal.

The ionic complexes PAA(MSS) were synthesized by the counterion exchange method; aqueous solutions of PAA and MSS were mixed in 1:1 stoichiometric ratio (PAA repeat units to MSS supramolecules), which resulted in the precipitation of the 1:1 complex. ${ }^{20}$ The MSSs bore terminal amine groups, and thus had to first be dissolved in acetone and converted to the ammonium chloride by addition of 1 equiv. of $\mathrm{HCl}$. The acetone solution was then diluted with 20 volumes of water and the complexation procedure initiated. For MSS1 and MSS2, 1:1 complexes were easily obtained. However, MSS3, unlike MSS1 and MSS2, showed slow precipitation from its aqueous solution, and therefore complexes with MSS3 could not be obtained.

For analysis by X-ray scattering, all samples were recast from chloroform solution and further annealed for 1 day in saturated chloroform vapor, followed by drying in a vacuum. No peaks were observed at wide angles for any of the samples, indicating no crystallinity of the alkyl tails (data not shown). The scattering patterns at small angles for the five samples are shown in Figure 1a. All these samples are mesomorphic, each showing two clear peaks in the SAXS. Of particular note is the striking qualitative similarity between SAXS results for MSP1 and PAA(MSS1), and between those for MSP2 and PAA(MSS2). Clearly, the ionic complexation of PAA and the MSS1 and MSS2 proceeded without interference from the bulkiness or charged nature of the $\mathrm{Ru}(\text { tpy })_{2}$ complexes, and the ionic + metal-coordination complexes show very similar morphology to their analogous MSP.

Table 1 summarizes the peak positions observed in SAXS, the corresponding repeat distances in real space, the ratio of the second peak position to the first $\left(q_{2} / q_{1}\right)$, and the estimated volume fraction of the $(1,2$, or 3$)$ alkyl tails. Unambiguous assignment of morphology from these SAXS results alone

(20) The 1:1 stoichiometry of the PAA(MSS) complexes could not be easily checked, so we also made complexes of MSS and a block copolymer of poly(ethylene oxide) (PEO)and poly(methacrylic acid). In this case, we could check the ratio of $\mathrm{O}-\mathrm{CH}_{2}$ protons from the $\mathrm{PEO}$ to $-\mathrm{CH}_{3}$ protons from the MSSs by proton NMR. In these cases, 1:1 complexation (MSS to acid groups) was observed for the MSS1 and MSS2. 
Table 1. Characteristics of MSP and PAA(MSS) Complexes

\begin{tabular}{lcccc}
\hline \multicolumn{1}{c}{ sample } & $q_{1}(1 / \mathrm{nm})$ & repeat dist. $(\mathrm{nm})$ & $\left(q_{1} / q_{2}\right)$ & $\begin{array}{c}\text { alkyl tail } \\
\text { vol. fr. }(\%)^{a}\end{array}$ \\
\hline MSP3 & 1.04 & 6.0 & $\sim \sqrt{ } 3$ & 31 \\
MSP2 & 1.13 & 5.6 & $\sim \sqrt{ } 3$ & 23 \\
MSP1 & 1.03 & 6.1 & $\sim 2$ & 14 \\
PAA(MSS2) & 1.06 & 5.9 & $\sim \sqrt{ } 3$ & 22 \\
PAA(MSS1) & 0.92 & 6.8 & $\sim 2$ & 14 \\
Tew-MSP-1xC16 $^{b}$ & 1.25 & 5.1 & $\sim \sqrt{ } 3$ & 20
\end{tabular}

${ }^{a}$ Volume fractions estimated using $\rho_{\text {tails }}=0.85, \rho_{\text {matrix }}=1.05^{b}$ From Aamer and Tew. ${ }^{9}$

is not straightforward; the positions of the second peaks relative to the first ones suggest hexagonal columnar morphology for the materials with 2 and 3 alkyl tails (peaks in a ratio of $q_{2} / q_{1} \approx \sqrt{ } 3$ ) and lamellar morphology for the 1-tail materials $\left(q_{2} / q_{1} \approx 2\right)$, however some questions regarding interpretation of the results remain. First of all, the peaks observed are all quite broad, and the "second-order" peaks are even broader than the 'first order' ones. In our reporting of $q_{2} / q_{1}$ in Table 1 , we wish to emphasize the approximate nature of the data. It would be a stretch, for example, to attempt to discern between hexagonal or distorted hexagonal (e.g., lattice parameters $a \neq b$ or $\theta \neq 120^{\circ}$ ) packing of columns in the cases where $q_{2} / q_{1} \approx \sqrt{ } 3$. Second, in the cases of the MSP1 and PAA(MSS1) samples, the intensity of the second peak is actually greater than that of the first. We know of no reports of lamellar systems in which this is the case, and can think of no circumstances under which it should be so. A more reasonable interpretation of the data for the onetail samples is a (poorly ordered) rectangular columnar arrangement ( $P 2 m$ symmetry $)^{21}$ with $a \approx 2 b$. When comparing the various samples, it is perhaps not surprising that the ordering should improve upon increasing the volume fraction of alkyl tails as in MSP1 $\rightarrow$ MSP2 $\rightarrow$ MSP3 (see Table 1). Here we also note that the SAXS pattern obtained by Aamer and Tew for their mesomorphous MSP is qualitatively similar to that of our MSP2, which is unsurprising given the similar alkyl tail volume fraction for these two samples.( $20 \%$ vs $\sim 23 \%$, respectively).

For additional morphological information, cross-polarized optical microscopy (CPOM) was performed on samples of MSP1, MSP2, and MSP3. Samples prepared by simple solvent casting showed weak birefringence. However, strong birefringence was observed for each of the MSPs if the samples were prepared by shearing a drop of viscous, highly concentrated solution and subsequent drying. Figure $1 \mathrm{~b}$ shows a representative image from the MSP2 sample obtained by this shearing procedure. The red color is due to the strong absorption of the metal-to-ligand charge-transfer (MLCT) band of the Ru(tpy) $)_{2}$ complex, ${ }^{8}$ and areas of differing brightness are due to areas of different sample thickness. Several cracks in the samples are also visible; for example one runs along the right edge of the image. Images from the MSP1 and MSP3 samples (shown in the Supporting Information) are qualitatively identical to those for MSP2. The CPOM results indicate that all three MSP samples adopt some optically anisotropic morphology, but with very short

(21) Canilho, N.; Kasemi, E.; Schluter, A. D.; Mezzenga, R. Macromolecules 2007, 40, 2822-2830.

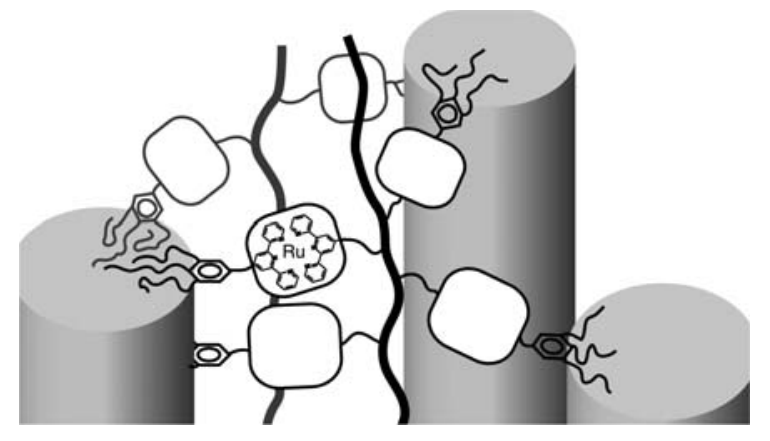

Figure 2. Schematic drawing of the proposed MSP3 morphology, in which the $\mathrm{C} 12$ alkyl tails form the cores of the dispersed columnar phase, whereas the polymer backbone and ruthenium bisterpyridine groups from the continuous matrix.

(less than optical wavelengths) correlation lengths unless some procedure is followed to globally orient the domains (e.g., the shearing procedure). These observations are consistent with our tentative assignment from the SAXS data of columnar morphologies.

For such columnar morphologies, the question arises as to which microphase-alkyl tails or backbone/bis(tpy) complex-composes the dispersed phase (the columns) and which composes the continuous matrix. Scattering methods, according to the "Babinet Principle," do not allow direct identification of domains but only the signature of the (liquid) crystalline space group in reciprocal space. ${ }^{22,23}$ Tew proposed a model wherein the $\mathrm{C} 16$ alkyl tails of his metallosupramolecular polymers formed the continuous phase, based on the assumption of the preservation of the very long persistence length of the polymer backbone observed in concentrated solution (the origin of the lyotropic liquid crystalline character observed in such solutions) even after solvent removal. This is indeed perhaps the most intuitive model. On the other hand, we believe the case is more likely to be reversed, with the alkyl tails composing the dispersed phase, as cartooned schematically in Figure $2 .{ }^{24}$ In support of this conjecture, we first note that the samples are very brittle, whereas a material composed of a continuous phase of (noncrystalline in the case of our C12 tails) alkyl tails should in theory be soft or waxy. Second, we note that the very small volume fraction occupied by the alkyl tails should promote confinement of the alkyl tails in the discrete domains in order to minimize total interfacial area (see Table 1). Recent studies involving real-space investigation of polyelectrolyte-surfactant complexes based upon dendrons, dendrimers, and dendronized polymers have shown cases where these rather nonintuitive configurations (i.e., columnar

(22) Spalla, O. In Neutrons, X-Rays and Light: Scattering Methods applied to Soft Condensed Matter; Lindner, P., Zemb, T., Eds.; Elsevier: Amsterdam, 2002; pp 49-71.

(23) We have attempted real-space imaging of these materials by transmission electron microscopy, but reliable images have to this point been unattainable, perhaps because of poor ordering (witness the relatively broad peaks observed in the SAXS data), the difficulty in obtaining proper staining conditions (high volume fraction of the stainable phase), and the low length scales involved.

(24) The C16 alkyl tails of the material reported by Tew show some semicrystallinity, in contrast to the shorter C12 tails reported here. This difference, however, should have little bearing on the topological arguments regarding the composition of the dispersed and continuous domains. 
phases where the columns are made up by the alkyl tail-rich phase) do indeed occur. ${ }^{25,26}$

In summary, we have shown that a series of polymers with one, two, or three $\mathrm{C} 12$ alkyl tails attached to the side-chain via metallosupramolecular chemistry form columnar liquid crystalline structures in the solid state, with the mesomorphic order increasing with the increasing alkyl tail content.

(25) Canilho, N.; Kasëmi, E.; Schlüter, A. D.; Ruokolainen, J.; Mezzenga, R. Macromolecules 2007, 40, 7609-7616.

(26) Mezzenga, R.; Ruokolainen, J.; Canilho, N.; Kasëmi, E.; Schlüter, A. D.; Lee, W. B.; Fredrickson, G. H. Soft Matter 2009, 5, 92-97.
Additionally, it is possible to achieve materials with similar morphology via an ionic supramolecular complexation between a simple polyelectrolyte and a metallosupramolecular surfactant.

Supporting Information Available: Experimental details, including information on MSP and MSS synthesis, and CPOM images from MSP1 and MSP3 (PDF). 